\title{
Pancreatic pleural effusion due to ductal disruption upstream of a tight ductal stricture in patient with chronic calcific pancreatitis: Successful management with pancreatic sphincterotomy and dilatation of ductal stricture by Guide Wire
}

\author{
Vishal Sharma, K. V. Raghavendra Prasada, Harish Kancharla, Ravi Sharma, Surinder S. Rana, \\ Deepak K. Bhasin
}

Department of Gastroenterology, Postgraduate Institute of Medical Education and Research, Sector 12, Chandigarh, India

\begin{abstract}
Endoscopic therapy has evolved as the standard of care for pancreatic pleural effusion and pancreatic ascites. Endoscopic retrograde cholangiopancreatography and bridging the disruption of ductal disruption with stent placement is the treatment of choice. However, it may not be always possible to negotiate tight pancreatic duct (PD) strictures or stricture stone complex, and endoscopic sphincterotomy alone may not be sufficient. We report a 53-year-old male who had chronic calcific pancreatitis with bilateral pancreatic pleural effusion and a tight stricture at head body junction, across which conventional endoscopic accessories could not be negotiated except for the 0.035 inch guidewire, which we kept across the stricture for $48 \mathrm{~h}$ for guidewire induced stricture dilation. This led to the complete resolution of symptoms and pancreatic pleural effusion. Combination of endoscopic sphincterotomy and guide wire induced stricture dilation can be used as rescue technique in cases of very tight PD strictures with complications such as pancreatic pleural effusion.

Key words Duct disruption, endoscopic sphincterotomy, ethanol, pancreatic ducts, guidewire, pancreatic stricture, pancreatitis, pleural effusion
\end{abstract}

\section{Introduction}

Pancreatic pleural effusion resulting from internal pancreatic fistulae is a well-recognized complication of chronic pancreatitis and may be difficult to manage. Pancreatico-pleural fistula (PPF) is seen in upto $0.4 \%$ cases of chronic pancreatitis and $4.5 \%$ patients of pancreatic pseudocysts. ${ }^{[1,2]}$ The underlying

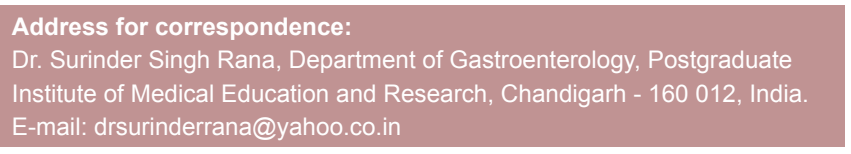

\begin{tabular}{|l|c|}
\hline \multicolumn{2}{|c|}{ Access this article online } \\
\hline \multirow{2}{*}{$\begin{array}{l}\text { Website: } \\
\text { www.jdeonline.in }\end{array}$} & Quick Response Code \\
\hline DOI: & \\
10.4103/0976-5042.159239 & \\
&
\end{tabular}

mechanism is usually a leak from an incompletely formed or a ruptured pseudocyst and around $10 \%$ patients have direct pancreatic duct (PD) leaks without any pseudocysts. ${ }^{[3,4]}$ The clinical consequences of PD disruption depends on several factors including the etiology, site of disruption, rate of secretion of pancreatic juice, location of the leak relative to anatomic tissue planes, ability of the systemic inflammatory response in containing the leak, and the presence of downstream obstruction of the PD by strictures/calculi ${ }^{[5,6]}$ If the duct disruption is anterior or if the pseudocyst leaks into the

This is an open access article distributed under the terms of the Creative Commons Attribution-NonCommercial-ShareAlike 3.0 License, which allows others to remix, tweak, and build upon the work non-commercially, as long as the author is credited and the new creations are licensed under the identical terms.

For reprints contact: reprints@medknow.com

How to cite this article: Sharma V, Raghavendra Prasada KV, Kancharla $\mathrm{H}$, Sharma R, Rana SS, Bhasin DK. Pancreatic pleural effusion due to ductal disruption upstream of a tight ductal stricture in patient with chronic calcific pancreatitis: Successful management with pancreatic sphincterotomy and dilatation of ductal stricture by Guide Wire. J Dig Endosc 2015;6:66-9. 
peritoneal cavity, it results in pancreatic ascites and in cases of posterior ductal disruption into the retroperitoneum or leakage of pseudocyst into pleural cavity results in pleural effusion..$^{[3,4]}$

The traditional options for management of pancreatic pleural effusion include conservative medical therapy or surgery. However, conservative measures have limited success, and surgical management is associated with significant morbidity. ${ }^{[1-4]}$ Because of being minimally invasive with excellent results, pancreatic endoscopic therapy with transpapillary PD stent/nasopancreatic drain placement is considered as the treatment of initial choice for management of pancreatic effusions. ${ }^{[4]}$ Multiple case series have reported successful treatment of pancreatic effusions with endoscopic therapy. ${ }^{[5-8]}$ The key to successful management in these cases is the bridging of the ductal disruptions, as well as any downstream ductal strictures/stones. ${ }^{[3,4]}$ We report a difficult case of pancreatic pleural effusion wherein a guidewire, placed across the PD stricture for $48 \mathrm{~h}$, was used for the purposes of dilatation in a patient with a tight PD stricture not traversable with conventional dilators or catheters.

\section{Case Report}

A 53-year-old male chronic smoker and a chronic alcohol consumer presented with pain abdomen for 3 months and right sided chest pain for 15 days. On examination, the patient was tachypneic (respiratory rate of $32 / \mathrm{min}$ ). Respiratory system examination revealed reduced breath sounds in right interscapular, infrascapular, axillary, infra-axillary area, and left the infrascapular area with dullness on percussion. Cardiovascular and neurological examination were unremarkable. Chest radiograph revealed bilateral pleural effusion (right $>$ left). Investigations revealed normal fasting blood sugar, liver, and renal biochemical parameters. Serum amylase and lipase levels were $1359 \mathrm{U} / \mathrm{L}$ (Normal up to $100 \mathrm{U} / \mathrm{L}$ ) and $428 \mathrm{U} / \mathrm{L}$ (Normal up to $60 \mathrm{U} / \mathrm{L}$ ), respectively. Diagnostic thoracocentesis from both sides revealed an exudative pattern with an amylase level of $>20,000 \mathrm{IU} / \mathrm{L}$, negative for malignant cells and acid fast bacilli and no organisms were cultured. Contrast-enhanced computerized tomography (CECT) of the chest and abdomen demonstrated features of chronic calcific pancreatitis with multiple pseudocysts in the lesser sac, as well as a peri-pancreatic region and bilateral pleural effusion [Figures 1 and 2]. Endoscopic ultrasound revealed pleural effusion with features suggestive of chronic calcific pancreatitis and strain ratio of 66.6 on elastography [Figures 3 and 4]. On endoscopic retrograde pancreatography (ERP) selective PD cannulation was achieved and minimal contrast was injected. Pancreatogram revealed a tight stricture with an upstream stone in the neck region and a disruption in the mid body level. The 0.035 inch guide wire (Jagwire; Boston Scientific) could be negotiated across the stricture, as well as disruption with difficulty. However, none of the accessories including the ERP cannula and dilators could be negotiated across the tight stricture [Figures 5 and 6]. Thereafter, a pancreatic sphincterotomy was done over the guide wire, and the guide wire was left in situ for $48 \mathrm{~h}$ [Figure 7]. Thereafter, the guide wire was removed and following this, the patient's symptoms gradually resolved. Repeat chest and abdomen CECT performed after 3 weeks revealed the complete resolution of

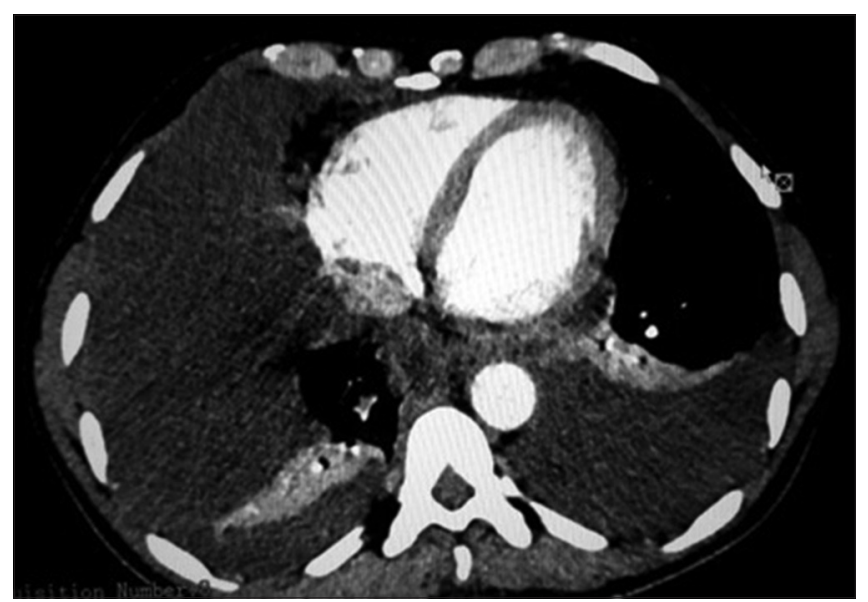

Figure 1: Computerized tomography image showing bilateral pleural effusion

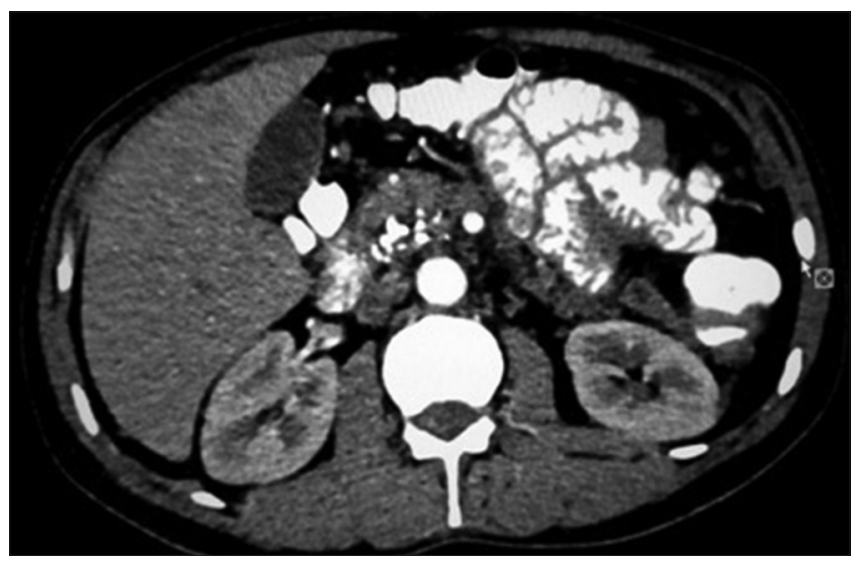

Figure 2: Computerized tomography showing multiple ductal calculi in the region of head

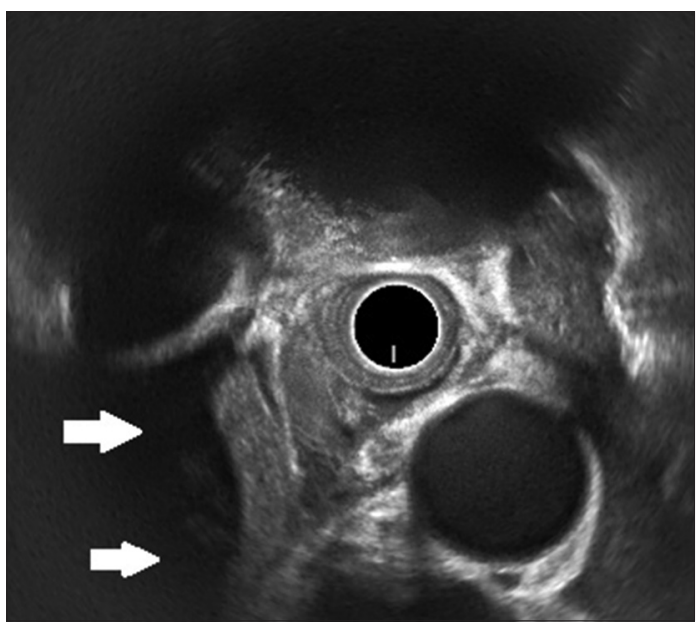

Figure 3: Endoscopic ultrasound showing right pleural effusion (arrow) 


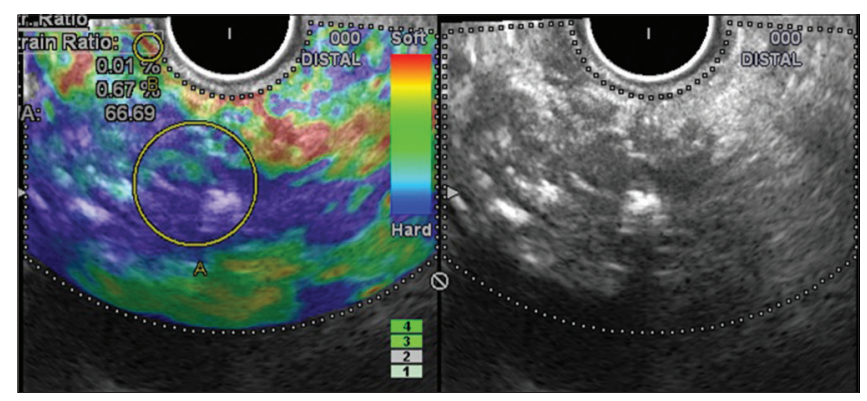

Figure 4: Endoscopic ultrasound elastography. Strain ratio of 66.6 has been calculated

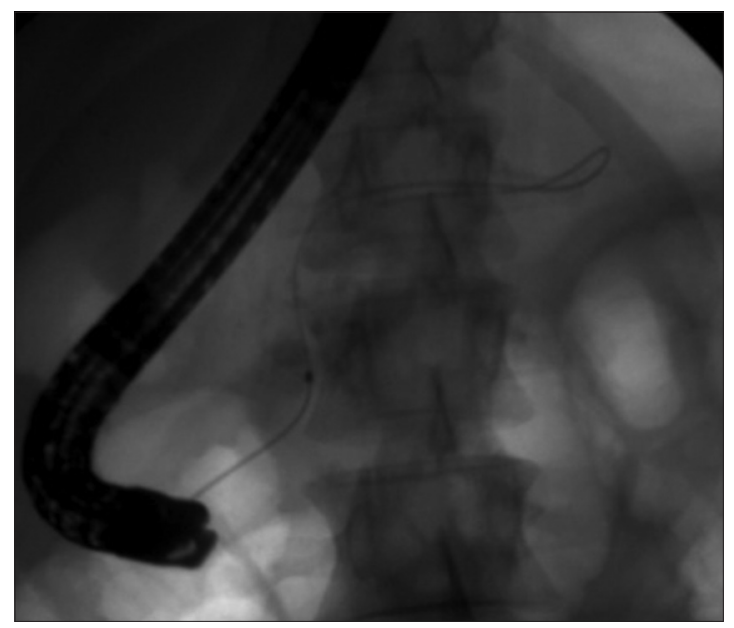

Figure 6: Guide wire negotiated across stricture and disruption

the bilateral pleural effusion. The patient is clinically well during the outpatient follow-up after six weeks, with no signs suggesting a recurrence on physical examination.

\section{Discussion}

Pleuro-pulmonary complications of pancreatitis include atelectasis, pleural effusion, acute respiratory distress syndrome, mediastinal pseudocysts, and PPF. The common symptoms of pancreatic pleural effusion include dyspnea, chest pain, cough, fever, and septicemia. ${ }^{[1-4]}$ A high index of suspicion is an important especially when there is massive effusion and recurring effusion after thoracentesis. Pancreatic pleural effusion occurs most commonly on the left side (51\%) followed by the right side (32\%) and less commonly they are bilateral $(16 \%){ }^{[1]}$

The available treatment modalities are conservative/medical that include octreotide and thoracentesis; endoscopic retrograde cholangiopancreatography (ERCP) with endoscopic pancreatic stent placement; and surgery. ${ }^{[4,5,7,8]}$ Conservative management includes keeping patients nil orally, total parenteral nutrition, and somatostatin analogs. The conservative treatment has a high failure rate of $50 \%$ and is complicated by malnutrition, central venous catheter infections, deep vein thrombosis, and sepsis associated with intestinal mucosal atrophy from

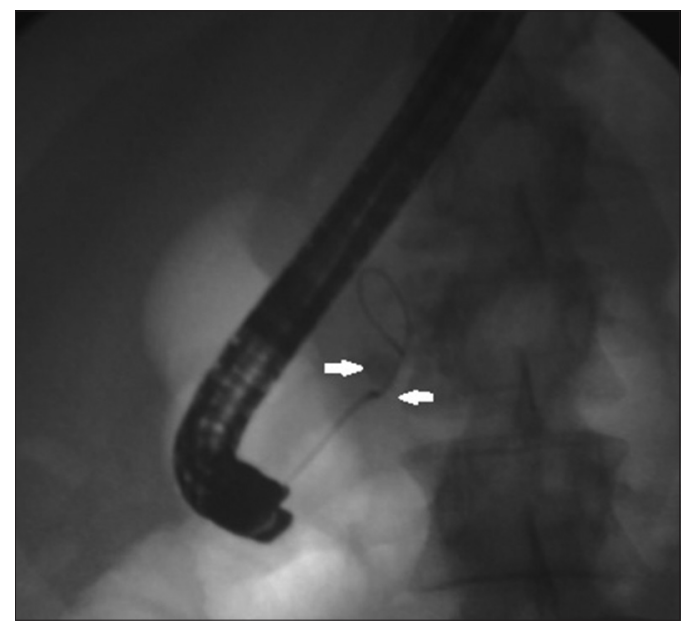

Figure 5: Endoscopic retrograde pancreatography image is showing stone at the head-body junction (arrow) and guidewire being negotiated across it. Note that cannula could not be taken across the narrowing

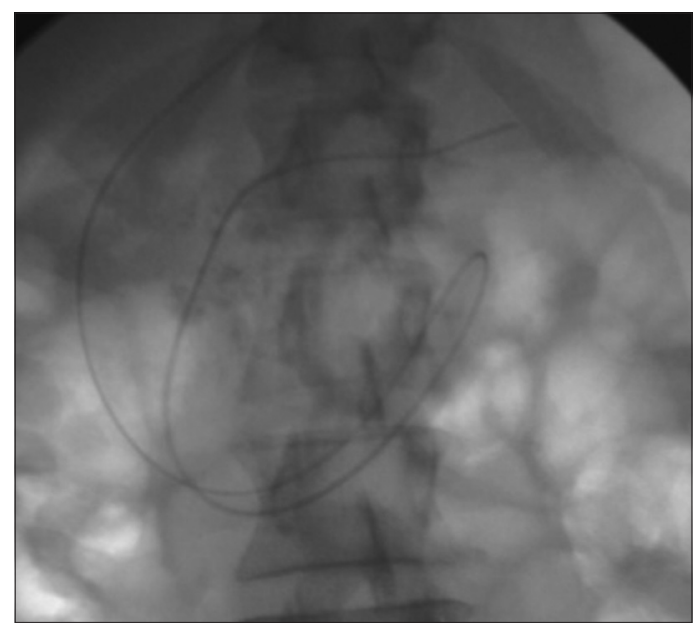

Figure 7: Guide wire left in situ for $48 \mathrm{~h}$ to allow for dilatation

prolonged fasting. ${ }^{[1-4]}$ With the advancements in endoscopic treatment, it has become first-line treatment in almost all such cases. ${ }^{[4]}$ ERCP is an effective management option with a low morbidity and mortality. ERCP facilitates pancreatic sphincterotomy, dilatation of main pancreatic duct (MPD) strictures, and extraction of stones from MPD with or without extracorporeal lithotripsy. ERCP and placement of either nasopancreatic drain (NPD) or stent across the leak/disruption is the usual method of choice. NPD can be used alone to treat pancreatic pleural effusion and ascites. ${ }^{[7]} \mathrm{NPD}$ is cumbersome for the patient and requires prolonged hospital stay though it has the advantage of taking repeat pancreatograms and can be flushed following blockage by necrotic/solid debris. ${ }^{[7]}$ Patients with partial duct disruption have a better outcome than complete disruption with endoscopic treatment. ${ }^{[7-11]}$

The key to successful management in these cases is the bridging of the ductal disruptions, as well as any downstream ductal strictures/stones. ${ }^{[3,4]}$ However, at times it may be difficult to traverse tight PD strictures or stricture with 
stones. In such cases, pancreatic sphincterotomy alone is less frequently successful. ${ }^{[12]}$ In our case, we left the 0.035 " guide wire (Jagwire; Boston Scientific) inside the PD for $48 \mathrm{~h}$ for facilitating the dilation of the tight stricture, as well as promote the healing of the PD disruption. The use of guidewire left across the stricture for $24 \mathrm{~h}$ for dilation of the stricture has been reported only rarely. ${ }^{[13]}$ The guidewire induced movements, as well as respiration induced movements, have been postulated to be a possible mechanism for inducing dilation. ${ }^{[13]}$ Our patient improved only with pancreatic sphincterotomy and guidewire induced dilation of the stricture, and there was a resolution of bilateral pancreatic pleural effusion. This technique can be an option in very tight pancreatic strictures not amenable to dilatation with conventional dilators as these may not traverse the extremely tight strictures. The guidewire dilatation would make the strictures permeable although they still may be non-negotiable as was in our case. However, the benefit of the technique is at the cost of additional discomfort to the patient.

\section{Financial support and sponsorship \\ Nil.}

\section{Conflict of interest}

There are no conflict of interest.

\section{References}

1. Oh YS, Edmundowicz SA, Jonnalagadda SS, Azar RR. Pancreaticopleural fistula: Report of two cases and review of the literature. Dig Dis Sci 2006;51:1-6.

2. Altasan T, Aljehani Y, Almalki A, Algamdi S, Talag A, Alkattan K.
Pancreaticopleural fistula: An overlooked entity. Asian Cardiovasc Thorac Ann 2014;22:98-101.

3. Dhebri AR, Ferran N. Nonsurgical management of pancreaticopleural fistula. JOP 2005;6:152-61.

4. Varadarajulu S, Rana SS, Bhasin DK. Endoscopic therapy for pancreatic duct leaks and disruptions. Gastrointest Endosc Clin N Am 2013;23:863-92.

5. Varadarajulu S, Noone TC, Tutuian R, Hawes RH, Cotton PB. Predictors of outcome in pancreatic duct disruption managed by endoscopic transpapillary stent placement. Gastrointest Endosc 2005;61:568-75.

6. Kozarek RA. Endoscopic therapy of complete and partial pancreatic duct disruptions. Gastrointest Endosc Clin N Am 1998;8:39-53.

7. Bhasin DK, Rana SS, Siyad I, Poddar U, Thapa BR, Sinha SK, et al. Endoscopic transpapillary nasopancreatic drainage alone to treat pancreatic ascites and pleural effusion. J Gastroenterol Hepatol 2006;21:1059-64.

8. Bhasin DK, Rana SS, Rao C, Gupta R, Verma GR, Kang M, et al. Endoscopic management of pancreatic injury due to abdominal trauma. JOP 2012;13:187-92.

9. Rana SS, Bhasin DK, Nanda M, Siyad I, Gupta R, Kang M, et al. Endoscopic transpapillary drainage for external fistulas developing after surgical or radiological pancreatic interventions. J Gastroenterol Hepatol 2010;25:1087-92.

10. Bhasin DK, Rana SS, Nanda M, Chandail VS, Masoodi I, Kang M, et al. Endoscopic management of pancreatic pseudocysts at atypical locations. Surg Endosc 2010;24:1085-91.

11. Bhasin DK, Rana SS, Udawat HP, Thapa BR, Sinha SK, Nagi B. Management of multiple and large pancreatic pseudocysts by endoscopic transpapillary nasopancreatic drainage alone. Am J Gastroenterol 2006;101:1780-6.

12. Bhasin DK, Malhi NS, Nagi B, Singh K. Pancreatic ascites treated by endoscopic pancreatic sphincterotomy alone: A case report. Gastrointest Endosc 2003;57:802-4.

13. Familiari P, Spada C, Costamagna G. Dilation of a severe pancreatic stricture by using a guidewire left in place for 24 hours. Gastrointest Endosc 2007;66:618-20. 